University of Nebraska - Lincoln

DigitalCommons@University of Nebraska - Lincoln

2013

\title{
Susceptibility of Fe atoms in Cu clusters
}

\author{
Rui Zhang \\ University of Nebraska-Lincoln, rzhang@huskers.unl.edu \\ Tom A. George \\ University of Nebraska-Lincoln \\ Parashu Kharel \\ University of Nebraska-Lincoln, pkharel2@unl.edu \\ Ralph Skomski \\ University of Nebraska-Lincoln, rskomski2@unl.edu \\ David J. Sellmyer \\ University of Nebraska-Lincoln, dsellmyer@unl.edu
}

Follow this and additional works at: https://digitalcommons.unl.edu/physicssellmyer

Zhang, Rui; George, Tom A.; Kharel, Parashu; Skomski, Ralph; and Sellmyer, David J., "Susceptibility of Fe atoms in Cu clusters" (2013). David Sellmyer Publications. 244.

https://digitalcommons.unl.edu/physicssellmyer/244

This Article is brought to you for free and open access by the Research Papers in Physics and Astronomy at DigitalCommons@University of Nebraska - Lincoln. It has been accepted for inclusion in David Sellmyer Publications by an authorized administrator of DigitalCommons@University of Nebraska - Lincoln. 


\title{
Susceptibility of Fe atoms in Cu clusters
}

\author{
Rui Zhang, ${ }^{\text {a) }}$ Tom A. George, Parashu Kharel, Ralph Skomski, and D. J. Sellmyer \\ Department of Physics \& Astronomy and Nebraska Center for Materials and Nanoscience, \\ University of Nebraska, Lincoln, Nebraska 68588, USA
}

(Presented 15 January 2013; received 5 November 2012; accepted 17 January 2013; published online 9 April 2013)

\begin{abstract}
By putting a Kondo system of limited dimension inside an insulating matrix, the Kondo screening cloud is confined by the size of the system. This allows us to distinguish the bulk and nano-particle behaviors of such system. We have investigated the magnetic properties of 0.3 at. \% Fe-doped $\mathrm{Cu}$ clusters with dimensions less than $20 \mathrm{~nm}$ embedded in a $\mathrm{SiO}_{2}$ matrix. The magnetic measurements are consistent with the Kondo interaction, and net antiferromagnetic Ruderman-Kittel-Kasuya-Yosida (RKKY) interactions between several Fe atoms in one cluster are estimated to have a very small effect. To understand the low-temperature susceptibility reduction, we compare Brillouin functions with exact quantum-mechanical solutions for interacting spin-1 particles and with the Kondo predictions for confined nanoparticles. (C) 2013 American Institute of Physics. [http://dx.doi.org/10.1063/1.4799617]
\end{abstract}

\section{INTRODUCTION}

The Kondo effect is normally associated with a lowtemperature resistivity minimum in magnetically doped metals. ${ }^{1}$ The phenomenon has been known for more than eighty years; ever since its discovery, it has become a testing ground for many numerical and analytical theories for the many-electron problem. ${ }^{2}$ However, the investigation of the phenomenon remains experimentally challenging due to the nature of the Kondo effect. It has recently attracted renewed attention leading to new insights into the problem. ${ }^{3}$

The basis of the Kondo effect is scattering and screening of conduction electrons by a magnetic impurity at low temperatures. Impurity spins in non-magnetic metals surround themselves with a cloud of screening electrons, the Kondo cloud. Aside from commonly used bulk resistivity measurements, several other experimental techniques used to investigate the Kondo effect include scanning tunneling microscopy ${ }^{4}$ and electrical measurements of quantum dots. ${ }^{5}$ In most studies, the Kondo cloud diameter is smaller than the dimensions of the investigated systems. As our previous study shows, ${ }^{3}$ even for nanoscale (or mesoscopic) Kondo effect studies, the conducting electrons can still travel beyond the borders of the Kondo cloud due to either the surrounding conducting matrix or the contacts required for such measurement. ${ }^{6}$ In nanoparticles, the conduction-electron states are discrete and the number of itinerant electrons contributing to the Kondo effect becomes smaller at low temperature. This alters the Kondo behavior and reduces the low-temperature magnetic susceptibility. ${ }^{3}$ Therefore, it is important to establish a system in which bulk and nanoscale Kondo effects can be distinguished.

The Kondo cloud can be restrained by putting magnetic impurities within an insulated metallic cluster with dimensions smaller than that of the Kondo cloud. ${ }^{3}$ Although resistivity measurements are difficult to perform for such a

\footnotetext{
a) Author to whom correspondence should be addressed. Electronic mail: rzhang@huskers.unl.edu
}

system, we can investigate the Kondo effect by studying the magnetic properties of such clusters.

\section{SAMPLE PREPARATION AND CHARACTERIZATION}

The clusters were generated in a home-made cluster-deposition system using DC magnetron sputtering in an $\mathrm{Ar} / \mathrm{He}$ environment after achieving a base pressure of $8 \times 10^{-8}$ Torr. $^{7}$ A 3-in. composite target was designed to give an iron concentration of 0.3 at.\% in the $\mathrm{Cu}(\mathrm{Fe})$ clusters. The gas aggregation chamber, where the clusters condensed in-flight, was cooled by liquid nitrogen. A pressure differential between the aggregation and deposition chambers drove the clusters through a $7 \mathrm{~mm}$ orifice and onto a substrate at ambient temperature. An insulating matrix of $\mathrm{SiO}_{2}$ was sputtered simultaneously onto the substrate from within the deposition chamber to ensure isolation of the individual copper clusters. The substrate normal was tilted $20^{\circ}$ from the cluster incidence and $70^{\circ}$ from the $\mathrm{SiO}_{2}$ particle beam for optimal co-deposition. Unless specified otherwise, cluster layers are reported in nominal thickness throughout this paper. The cluster size distribution was determined by transmission electron microscopy (TEM) from a $\mathrm{Cu}(\mathrm{Fe})$ cluster layer covered by $\mathrm{SiO}_{2}$ deposited on a copper grid.

Magnetic measurements were performed on a sample consisting of approximately $16 \%$ volume percent of $\mathrm{Cu}(\mathrm{Fe})$ clusters in a $780 \mathrm{~nm}$ thick $\mathrm{SiO}_{2}$ matrix on a Kapton film substrate. The $1 \mathrm{in}$. square flexible substrate was then folded into a hollow cylinder of $3 \mathrm{~mm}$ height and $5 \mathrm{~mm}$ diameter for measurement in a superconducting quantum interference device (SQUID) magnetometer. The zero field cool (ZFC) measurement covered the temperature range of $2 \mathrm{~K}$ to $150 \mathrm{~K}$ with an applied field of 200 Oe. For comparison, a thin film sample of $50 \mathrm{~nm}$ of the same $\mathrm{Cu}(\mathrm{Fe})$ was sputtered on a $\mathrm{Si}$ substrate. The resistivity of that sample was measured from $2 \mathrm{~K}$ to 300 K with a Physical Property Measurement System (PPMS) to estimate the Kondo temperature of an analogous bulk system.

\section{RESULTS AND DISCUSSION}

The film sample prepared for resistivity measurement shows clear Kondo behavior as shown in Fig. 1. As temperature 


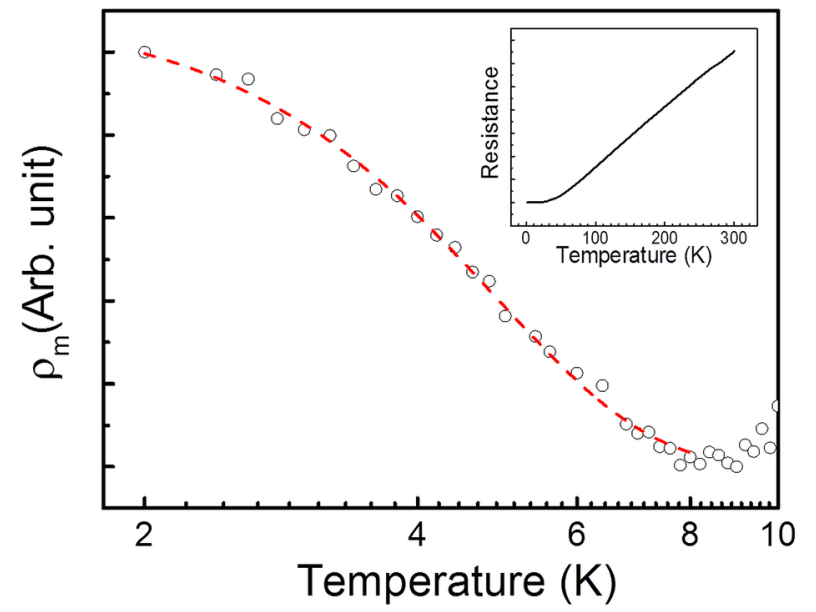

FIG. 1. Temperature dependence of resistivity of the film sample $\rho_{\mathrm{m}}=\rho-$ $\rho_{\text {min }}$ between $2 \mathrm{~K}$ and $11 \mathrm{~K}$. The dashed line represents the NRG fitting. The inset shows resistivity as a function of temperature measured between $2 \mathrm{~K}$ and $300 \mathrm{~K}$.

decreases from $300 \mathrm{~K}$, the resistivity drops linearly with the decreasing temperature. However, a resistivity minimum followed by a logarithmic temperature dependence is observed below $10 \mathrm{~K}$. As the temperature decreases further, the resistivity shows a tendency towards saturation. The measured result was fitted using the numerical renormalization-group (NRG)like equation $^{8}$

$$
\rho_{m}=\rho_{0}\left[1+\left(2^{\frac{1}{\alpha_{s}}}-1\right)\left(\frac{T}{T_{K}}\right)^{\xi_{s}}\right]^{-\alpha_{s}}
$$

where $\rho_{0}$ is the residual resistivity, $T_{\mathrm{K}}$ is the Kondo temperature, and $\xi_{\mathrm{s}}$ and $\alpha_{\mathrm{s}}$ are fitting parameters. ${ }^{9}$ As shown in Fig. 1, a reasonably good fit can be achieved using the above equation and the $T_{K}$ acquired from the fit is $4.2 \mathrm{~K}$. The Kondo screening length $\xi_{\mathrm{K}}$ can be estimated using the following equation: ${ }^{10}$

$$
\xi_{K}=\frac{\hbar v_{F}}{k_{B} T_{K}}
$$

where $v_{\mathrm{F}}=1.57 \times 10^{6} \mathrm{~m} / \mathrm{s}$ is the Fermi velocity of copper. ${ }^{11}$ Therefore, the estimated Kondo screening length is around $3 \mu \mathrm{m}$. As revealed by the TEM image in Fig. 2, the $\mathrm{Cu}(\mathrm{Fe})$ clusters have an average diameter of around $4 \mathrm{~nm}$, which is much smaller than the estimated Kondo-cloud screening length. The ZFC measurement for $\mathrm{Cu}(\mathrm{Fe})$ clusters embedded in a $\mathrm{SiO}_{2}$ matrix is shown in Fig. 3. The curve shows clear paramagneticlike behavior and no downturn was observed above $2 \mathrm{~K}$. It can be fitted reasonably well with the following expression:

$$
M=\chi_{0} H+\frac{C H}{T-\theta},
$$

where $\chi_{0}$ represents the temperature-independent term, while the second term is due to the impurities plus Kondo interactions. The fitting yields $\theta=-0.7 \mathrm{~K}$, which is smaller than the value acquired in bulk system, ${ }^{12}$ suggesting the Kondo interaction is suppressed by the reduced size of the system. The inset of Fig. 3 shows the $M(H)$ curve after subtracting

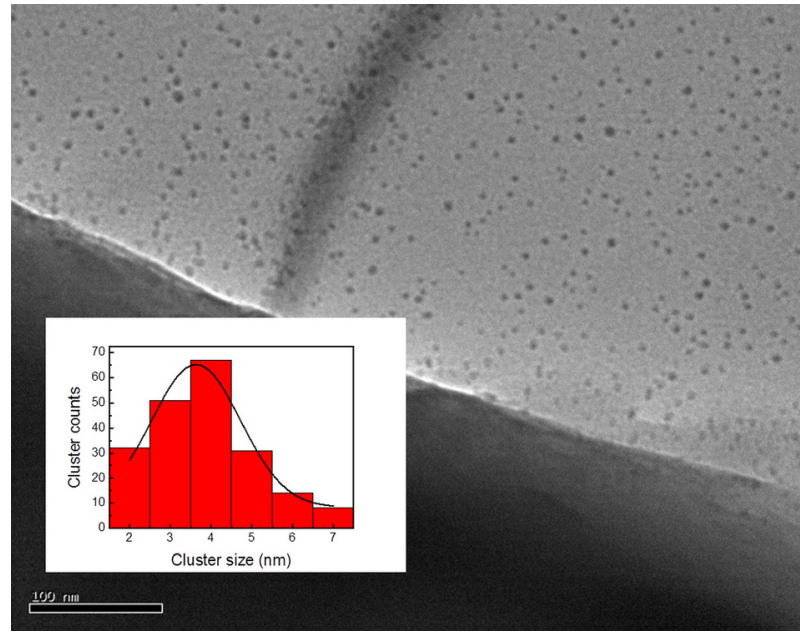

FIG. 2. TEM image of $\mathrm{Cu}(\mathrm{Fe})$ clusters deposited on TEM grid. The inset is the cluster size distribution measured from the sample.

the diamagnetic background, and a reasonable fit can be achieved using the Brillouin function with $S=1$.

Since the $\mathrm{Fe}$ atoms are randomly distributed in the $\mathrm{Cu}$ matrix, they are likely to experience coupling due to RKKY interactions. If these interactions are predominantly antiferromagnetic (AFM), then they may mimic or mask the Kondo effect, because both mechanisms reduce the low-temperature susceptibility with respect to the Curie 1/T law. The interaction effect must be treated quantum-mechanically, because the Kondo effect consists in the discrete flipping of individual spins, and such a quantum-mechanical flipping can also be caused by AFM interactions. A classical interaction would yield an unphysical continuous "wiggling" of the coupled spins and blur the discrete character of the Kondo effect.

For interacting spin-1 particles, the spin-1/2 Pauli matrices must be replaced by the spin operators ${ }^{13}$

$$
\begin{aligned}
& \mathrm{S}_{\mathrm{x}}=\frac{1}{\sqrt{2}}\left(\begin{array}{ccc}
0 & 1 & 0 \\
1 & 0 & 1 \\
0 & 1 & 0
\end{array}\right) ; \mathrm{S}_{\mathrm{y}}=\frac{\mathrm{i}}{\sqrt{2}}\left(\begin{array}{ccc}
0 & -1 & 0 \\
1 & 0 & -1 \\
0 & 1 & 0
\end{array}\right) ; \\
& \mathrm{S}_{\mathrm{z}}=\left(\begin{array}{ccc}
1 & 0 & 0 \\
0 & 0 & 0 \\
0 & 0 & -1
\end{array}\right) .
\end{aligned}
$$

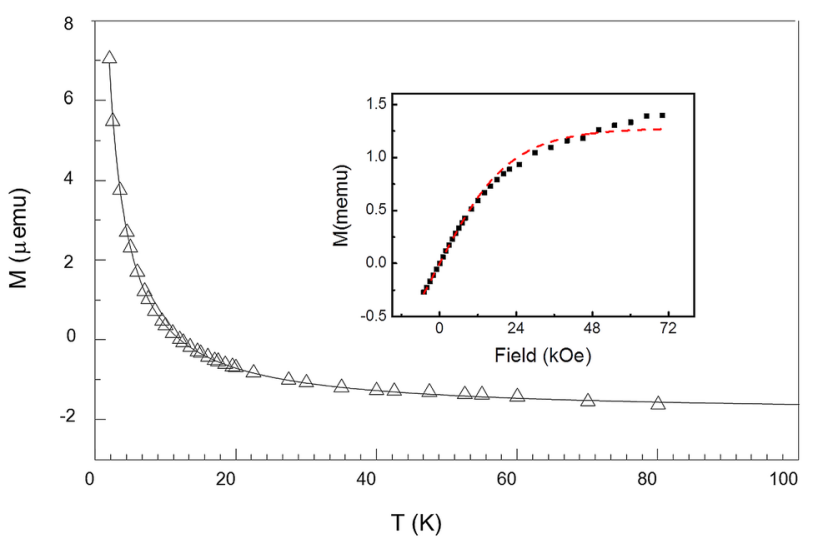

FIG. 3. ZFC measurement of $\mathrm{Cu}(\mathrm{Fe})$ clusters embedded in a $\mathrm{SiO}_{2}$ matrix. The solid line is the fit to the data. The inset is the $M$ vs. $H$ curve measured at $2 \mathrm{~K}$ and the dashed line is the Brillouin function fit to the data. 


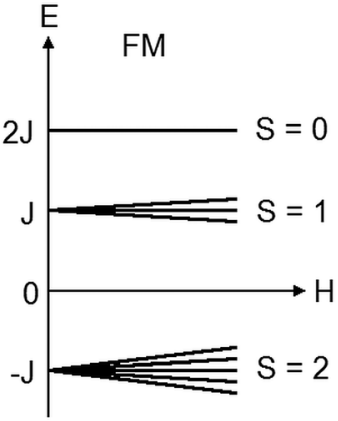

(a)

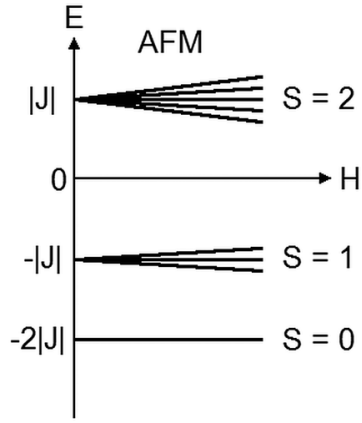

(b)
FIG. 4. Energy levels for two interacting $S=1$ atoms with (a) ferromagnetic and (b) AFM RKKY interaction.

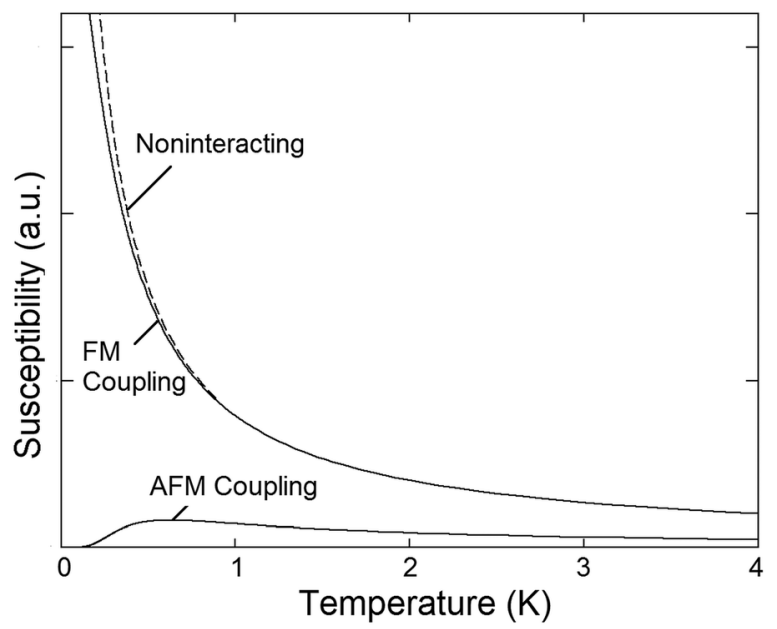

FIG. 5. Susceptibility of non-interacting and interacting $\mathrm{Fe}$ atoms in $\mathrm{Cu}$. Only pairs of $\mathrm{Fe}$ atoms interacting by positive or negative RKKY interactions are considered, and the susceptibility of the non-interacting system has been normalized to agree with the high-temperature part of the FM curve.

For isolated Fe particles in a field $\mathbf{H}=H \mathbf{e}_{\mathrm{z}}$, only the last matrix is important, and the susceptibility is given by the Brillouin function $B_{1}(x)$ where $x=\mathrm{g} \mu_{\mathrm{B}} \mathrm{S} / \mathrm{k}_{\mathrm{B}} T, S=1$. We consider pairs of $S=1$ spins $S_{1}$ and $S_{2}$, coupled by Heisenberg exchange $-J \mathrm{~S}_{1} \cdot \mathrm{S}_{2}$. The interactions can then be written as direct products of the matrices of Eq. (2) for $S_{1}$ and $S_{2}$. The diagonalization of the resulting $9 \times 9$ matrix yields an $S=2$ quintuplet of energy $-J$, an $S=1$ triplet of energy $+J$, and an $S=0$ singlet of energy $+2 J$. Fig. 4 shows these levels for positive (ferromagnetic (FM)) and negative (AFM) values of $J$.

In the ferromagnetic case, the main contribution to the susceptibility comes from the ferromagnetic quintuplet, bottom of Fig. 4(a), with small corrections due to the triplet. For antiferromagnetic coupling, the situation is more complicated. The AFM ground state $(S=0)$ does not contribute to the susceptibility, but the $S=1$ triplet is fairly close to the singlet and gives rise to a Van-Vleck-type susceptibility. Physically, the singlet and triplet states mean that the two coupled spin-1 atoms involve four spin-1/2 electrons with "two spins up, two spins down" (singlet) and "three spins up, one spin down" (triplet), and the corresponding wave functions are obtained by diagonalizing the above-mentioned $9 \times 9$ matrix.

The susceptibilities are readily obtained via the partition functions belonging to Figs. 4(a) and 4(b). In the FM case (a), the result is, in lowest order, equal to the susceptibility predicted by the Brillouin function $B_{2}\left(x^{\prime}\right)$ where $x^{\prime}=\mathrm{g} \mu_{\mathrm{B}} 2 \mathrm{~S} /$ $\mathrm{k}_{\mathrm{B}} T, S=1$. In the AFM case (b), the lowest-order susceptibility is zero, but there is a small Van-Vleck contribution proportional to the small parameter $\lambda=\exp \left(-|J| / \mathrm{k}_{\mathrm{B}} T\right)$. By contrast, the triplet correction to the FM quintuplet is of the order $\lambda^{2}=\exp \left(-2|J| / \mathrm{k}_{\mathrm{B}} T\right)$. Fig. 5 compares the corresponding susceptibility contributions. In the present case, the average $\mathrm{Fe}-\mathrm{Fe}$ distance is $1.6 \mathrm{~nm}$. Around this distance, the RKKY exchange is negative and small, less than about $0.5 \mathrm{~K}$ in temperature units, or positive, ${ }^{14}$ and should not interfere very much with the Kondo effect.

\section{SUMMARY}

In conclusion, we have observed the Kondo effect in dilute $\mathrm{Cu}: \mathrm{Fe}$ thin-film samples. Our magnetic susceptibility measurements indicate that the effect is reduced in isolated $\mathrm{Cu}: F e$ particles. This is ascribed to the strong reduction of the Kondo screening cloud, which cannot be bigger than the particle size, and consistent with theoretical predictions. RKKY interactions between Fe atoms in one cluster are estimated to yield very small corrections.

\section{ACKNOWLEDGMENTS}

This research was supported by NSF MRSEC (R.S.), and DOE (D.J.S.).

${ }^{1}$ J. Kondo, Prog. Theor. Phys. 32(1), 37 (1964).

${ }^{2}$ S. Smirnov and M. Grifoni, Phys. Rev. B 84, 235314 (2011).

${ }^{3}$ R. Skomski, R. Zhang, P. Kharel, A. Enders, S. H. Liou, and D. J. Sellmyer, J. Appl. Phys. 107, 09E126 (2010).

${ }^{4}$ N. Neel, J. Kroger, R. Berndt, T. O. Wehling, A. I. Lichtenstein, and M. I. Katsnelson, Phys. Rev. Lett. 101, 266803 (2008).

${ }^{5}$ W. Izumida and O. Sakai, J. Phys. Soc. Jpn. 74, 103 (2005).

${ }^{6}$ O. Ujsaghy and A. Zawadowski, J. Phys. Soc. Jpn. 74, 80 (2005).

${ }^{7}$ B. Balamurugan, B. Das, V. R. Shah, R. Skomski, X. Z. Li, and D. J. Sellmyer, Appl. Phys. Lett. 101, 122407 (2012).

${ }^{8}$ P. Kharel, R. Skomski, P. Lukashev, R. Sabirianov, and D. J. Sellmyer, Phys. Rev. B 84, 014431 (2011).

${ }^{9}$ J. J. Parks, A. R. Champagne, T. A. Costi, W. W. Shum, A. N. Pasupathy, E. Neuscamman, S. Flores-Torres, P. S. Cornaglia, A. A. Aligia, C. A. Balseiro, G. K. L. Chan, H. D. Abruna, and D. C. Ralph, Science 328, 1370 (2010).

${ }^{10}$ M. Ternes, A. J. Heinrich, and W. D. Schneider, J. Phys.: Condens. Matter 21, 053001 (2009).

${ }^{11}$ C. Kittel, Introduction to Solid State Physics, 8th ed. (Wiley, Hoboken, NJ, 2005).

${ }^{12}$ J. M. Franz and D. J. Sellmyer, Phys. Rev. B 8, 2083 (1973).

${ }^{13}$ D. J. Craik, Magnetism: Principles and Applications (Wiley, Chichester, NY, 1995).

${ }^{14}$ A. A. Khajetoorians, J. Wiebe, B. Chilian, S. Lounis, S. Blugel, and R. Wiesendanger, Nat. Phys. 8, 497 (2012). 